\title{
Wavlet Decomposition based Diagnostic for Structural Health Monitoring on Metallic Aircrafts: Case of Crack Triangulation and Corrosion Detection
}

\author{
Hamza Boukabache ${ }^{1}$, Christophe Escriba ${ }^{2}$, Sabeha Zedek ${ }^{3}$ and Jean-Yves Fourniols ${ }^{4}$ \\ ${ }^{1,2,4}$ C.N.R.S-LAAS French national center for scientific research, Toulouse, 31400, FRANCE \\ hboukaba@laas.fr \\ cescriba@laas.fr \\ fourniols@laas.fr \\ ${ }^{3}$ Université de Toulouse, INSA - Toulouse, 31400, France \\ sfzedek@laas.fr
}

\begin{abstract}
This work focus on the structural health monitoring of aircrafts parts specimen structures made of 2024 Aluminum alloys using a reliable Joint Time Frequency Analysis calculation (Joint Temporal Frequency Analysis). In this paper we demonstrate the feasibility of a new non destructive control method capable to probe very large structures within a short time. The method we developed is based through a wide piezoelectric sensors network on a smart comparison between two acoustic signatures: the healthy structure response captured before the commissioning of the plane and "an after flight" response. The sensors network exploits the capability of piezoelectric patches to generate/measure specific Lamb wave's modes. The system is therefore dynamically configured to localize mechanicals flaws using a triangulation algorithm that operates using different techniques like pitch-catch and pulse-echo. The aim of this paper is to highlight a methodology that is currently being integrated into reconfigurable qualified and certified hardware architecture. The idea behind is to interface the airplane's structure to an integrated modular avionics calculator (IMA).
\end{abstract}

An analytic study is performed and tests to prove the proposed method feasibility on corroded and damaged structures specimens are provided at the end of this paper.

\section{INTRODUCTION}

Damage detection and structural health monitoring is one of the major challenges that face aircraft manufacturers. With the

Hamza Boukabache et al. This is an open-access article distributed under the terms of the Creative Commons Attribution 3.0 United States License, which permits unrestricted use, distribution, and reproduction in any medium, provided the original author and source are credited. perpetual increase of structures complexity and the constant integration of composite materials to lighten aircrafts weight, the development of new non destructives tools capable to detect different flaws natures is more necessary than ever before.

Based on Eddy current (Henry A. Sodano, 2007), ultrasonic's radar (Victor Giurgiutiu, 2004) or thermal imaging (McIntosh, Greg, 1996) these widely developed and used techniques are locals, very accurate but definitely unsuitable to probe a complete aircraft structures in a reasonable laps of time.

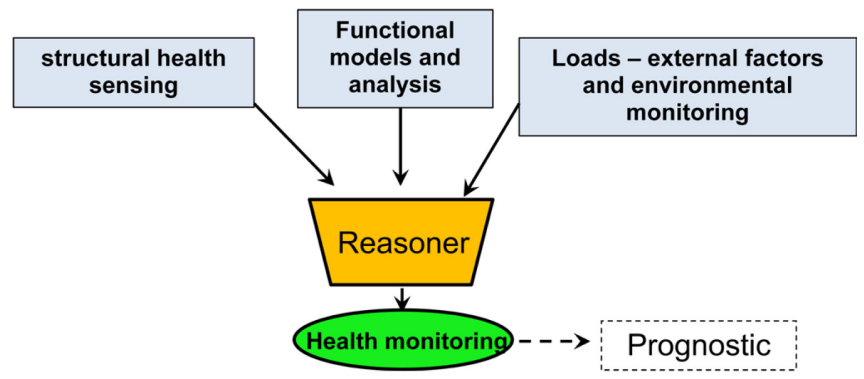

Figure 1. Synoptic of a complete PHM (Prognostics and Health Management) system for aircraft's structures.

(Courtesy of Eric Lindgren, Air Force Research Lab)

Some other promising works were performed on impact energy propagation (Salsabil Ksouri et al, 2011) through MEMS accelerometric sensors. Electromechanical (E/M) impedancebased structural health monitoring has shown quick success for detecting incipient damage using relatively cheap sensors. The concept was primarily used for non-destructive inspection by Liang (1994) and sun (1995). Recent research conducted by Airbus and the French C.N.R.S initiate the field integration of the method (Hamza Boukabache et al, 2012). 
Active health monitoring and non destructive evaluation (NDE) systems for aircraft structures using guided waves and more specifically Lamb waves are relatively new and modern. These waves are able to travel from a single excitation point through very large distances in the medium where they propagate within a minimum energy loss. So far, the exploitation of these waves were widely focused on plate-like structures and others simple geometries made of a unique material like oil pipe-line and (Dixon, $\mathrm{S}$ et al. 2004). Based on echoes identification some commercial applications (OLYMPUS, 2009) and DO-160 qualifiable tools (Seth S. Kessler et al, 2009) have therefore recently emerged for local inspection. However in more complex geometries, the multiple reflections due to the various structural features make the distinction between the different echoes quite difficult. Another problem emerges from the fact that Lamb wave are multimodal and dispersive by nature which makes the analysis very difficult in large area structures. Many studies were focused on a Lamb wave frequency tuning (Victor Giurgiutiu, 2005) to generate a pure mode or just to maximize/minimize a particular mode response. Although, this technique gives very good results in cracks detection for metal plate like structures, it seems to be unsuited for complex geometries which show a high thickness variation.

To circumvent these different problems, we propose a diagnosis method based on a smart comparison algorithm between two signatures: a healthy and a damaged one (Hamza Boukabache et $\mathrm{al}, 2011)$. The first signature is captured before the commission of the plane. The saved signals will be used in a database as a baseline for a future control. The signature of the structure is captured through a large sensors/actuators network that uses the capability of piezoelectric materials to generates/measures specific Lamb wave's modes (Hamza Boukabache et al, 2011). The different interaction of theses waves into the probed structures are therefore exploited to extract a significant signature. The diagnosis is thus based on a correlation between a baseline and a captured signature. This correlation is performed using a wavelet transform to have a time frequency comparison. The asset of this methodology is to have fast diagnostic and a quick localization of an eventual existing damage even in large area aircraft structures whatever the nature of the damage. Another advantage of the proposed method is its capability to detect corrosion (Hamza Boukabache et al, 2012) which represents one of the most critical damage on aerospace metallic structures. The detection of such flaw become a priority issue for the US Air Force (Dustin Thomas et al 2004) that spend 1.2 billion USD a year to repair corrosion on the KC-135 for example.

The work presented in this paper is the first step toward a complete PHM (Prognostic and Health Management) system presented in figure 1. The development of a reliable diagnosis method is discussed through the use of Lamb wave to extract a significant structure baseline. An analytical study is performed and correlated to the experimental results. The generation of these specifics waves using piezoelectric wafer is also studied and the results are confronted to the multiple issues linked to the bonding with the host structure.

\section{LAMB WAVES THEORY}

Guided waves have a major importance in SHM applications. In opposite to the reset of elastic waves, they can travel on longue distances and cover larges areas. The created acoustic compression field stays confined between the structure's faces if the wavelength of the propagating wave is comparable to the structure thickness.

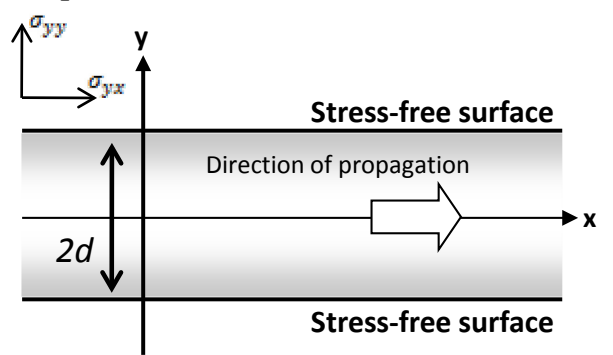

Figure 2. Plate-like structure with a thickness of $2 d$. The upper and lower surfaces are unconstrained and stressfree.

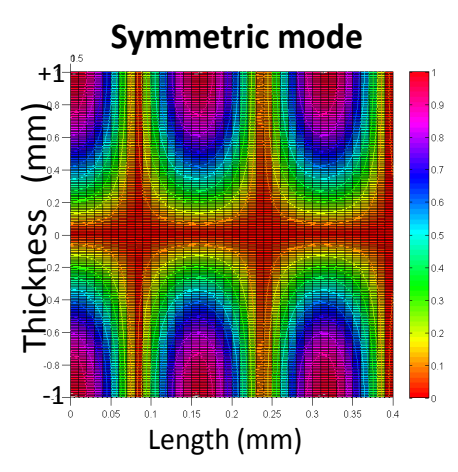

(a)

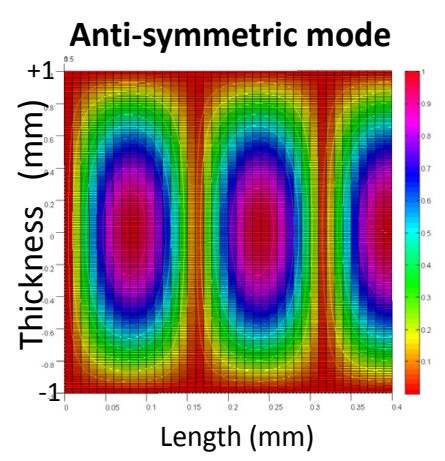

(b)
Figure 3. Numerical resolution of the deformation equation $\vec{u}(t)$. The excitation frequency is $200 \mathrm{kHz}$.

The acoustic compression field is simulated for a normalized structure thickness. (a) The simulation result for the fundamental symmetric mode $\mathrm{S}_{0}$ (b) The fundamental anti-symmetric motion mode $\mathrm{A}_{0}$.

For isotropic materials the study of these waves begins with the general equation of dynamics applied to elastics materials:

$$
\mu \nabla^{2} \overrightarrow{\boldsymbol{u}}+(\lambda+\mu) \nabla \nabla \cdot \overrightarrow{\boldsymbol{u}}=\rho \frac{\partial^{2} \boldsymbol{u}}{\partial t^{2}}
$$

Where $\overrightarrow{\boldsymbol{u}}$ is the deformation vector, $\lambda$ and $\mu$ are the Lame constants and $\rho$ is the mass density.

For plate-like isotropic structures, the symmetry of the geometry creates invariance in the orthogonal direction which allows using Helmholtz decomposition to reduce $\overrightarrow{\boldsymbol{u}}$ into a sum of a potential vector and a potential scalar.

$$
\overrightarrow{\boldsymbol{u}}=\nabla \Phi+\operatorname{curl} \overrightarrow{\boldsymbol{\Psi}}
$$


By developing the equation (1) using the relation (2) we find the differentials equations of elastic wave propagation.

$$
\begin{cases}c_{p}^{2} \Delta^{2} \Phi=\frac{\partial^{2} \Phi}{\partial t^{2} \rho} & \text { with } \quad c_{p}^{2}=\underline{(\lambda+2 \mu)} \\ c_{S}^{2} \Delta^{2} \overrightarrow{\boldsymbol{\Psi}}=\frac{\partial^{2} \overrightarrow{\boldsymbol{\Psi}}}{\partial t^{2} \rho} & \text { with } \quad c_{S}^{2}=\underline{\mu}\end{cases}
$$

With:

$$
\begin{gathered}
\Phi=\Phi(y) \cdot e^{i(k \cdot x-\omega t)} \\
\overrightarrow{\boldsymbol{\Psi}}=\left(\Psi_{x}(y) \overrightarrow{e_{x}}+\Psi(y) \overrightarrow{e_{y}}+\Psi_{z}(y) \overrightarrow{e_{z}}\right) \cdot e^{i(k \cdot x-\omega t)}
\end{gathered}
$$

Where, $\mathrm{k}$ is the wave number, $\omega$ is the frequency; $\Psi_{x}, \Psi_{y}, \Psi_{z}$ are the scalars components of $\overrightarrow{\boldsymbol{\Psi}}$. The substitution of the equations (5) and (3) in the system (3) gives four scalars differentials equations that accept solutions in the form:

$$
\left\{\begin{array}{l}
\Phi=(A \cdot \cos p y+H \cdot \sin p y) e^{i(k \cdot x-\omega t)} \\
\Psi_{x}=(B \cdot \cos q y+G \cdot \sin q y) e^{i(k \cdot x-\omega t)} \\
\Psi_{y}=(E \cdot \cos q y+D \cdot \sin q y) e^{i(k \cdot x-\omega t)} \\
\Psi_{z}=(C \cdot \cos q y+F \cdot \sin q y) e^{i(k \cdot x-\omega t)}
\end{array}\right.
$$

Where, $p^{2}=\omega^{2} / c_{p}^{2}-k^{2}$ et $q^{2}=\omega^{2} / c_{s}^{2}-k^{2}$ and $A, B$, $C, D, E, F, G, H$ are the integration constants. These constants are found only by setting the boundary conditions. We assume that structures thickness is equal to $2 d$ and its upper and lower surfaces are stress-free:

$$
\begin{gathered}
\sigma_{y x}(x,-d)=-\sigma_{y x}(x, d)=0 \\
\sigma_{y y}(x,-d)=\sigma_{y x}(x, d)=0
\end{gathered}
$$

In this case the integrations constants are given by the system of equations (8). Its complete resolution leads to the particle movement equation $\vec{u}(t)$.

The calculation of the coefficient matrix determinant yields to the characteristic equation of guided waves. This determinant is constituted by the smaller determinants couples $(A, B),(C, D),(E, F),(G, H)$ where each one specifies a different kind of motion. The two first couples correspond respectively to the symmetric and antisymmetric Lamb wave's modes and the rest pairs correspond to symmetric and anti-symmetric SH waves.

$$
\left(\begin{array}{cccccccc}
C_{11} & C_{12} & 0 & 0 & 0 & 0 & 0 & 0 \\
C_{21} & C_{22} & 0 & 0 & 0 & 0 & 0 & 0 \\
0 & 0 & C_{33} & C_{34} & 0 & 0 & 0 & 0 \\
0 & 0 & C_{43} & C_{44} & 0 & 0 & 0 & 0 \\
0 & 0 & 0 & 0 & C_{55} & C_{56} & 0 & 0 \\
0 & 0 & 0 & 0 & C_{65} & C_{66} & 0 & 0 \\
0 & 0 & 0 & 0 & 0 & 0 & C_{77} & C_{78} \\
0 & 0 & 0 & 0 & 0 & 0 & C_{87} & C_{88}
\end{array}\right)\left(\begin{array}{c}
A \\
B \\
C \\
D \\
E \\
F \\
G \\
H
\end{array}\right)=0
$$

With:

$$
\begin{array}{ll}
C_{11}=-2 i k p \sin p d & C_{12}=\left[k^{2}-q^{2}\right] \sin q d \\
C_{22}=2 i \mu k q \cos q d & C_{21}=\left[(\lambda+2 \mu) \alpha^{2}+\lambda k^{2}\right] \cos p d \\
C_{43}=2 i k p \cos p d & C_{44}=\left[k^{2}-q^{2}\right] \cos q d \\
C_{34}=-2 i \mu k q \sin q d & C_{33}=\left[(\lambda+2 \mu) \alpha^{2}+\lambda k^{2}\right] \sin p d
\end{array}
$$
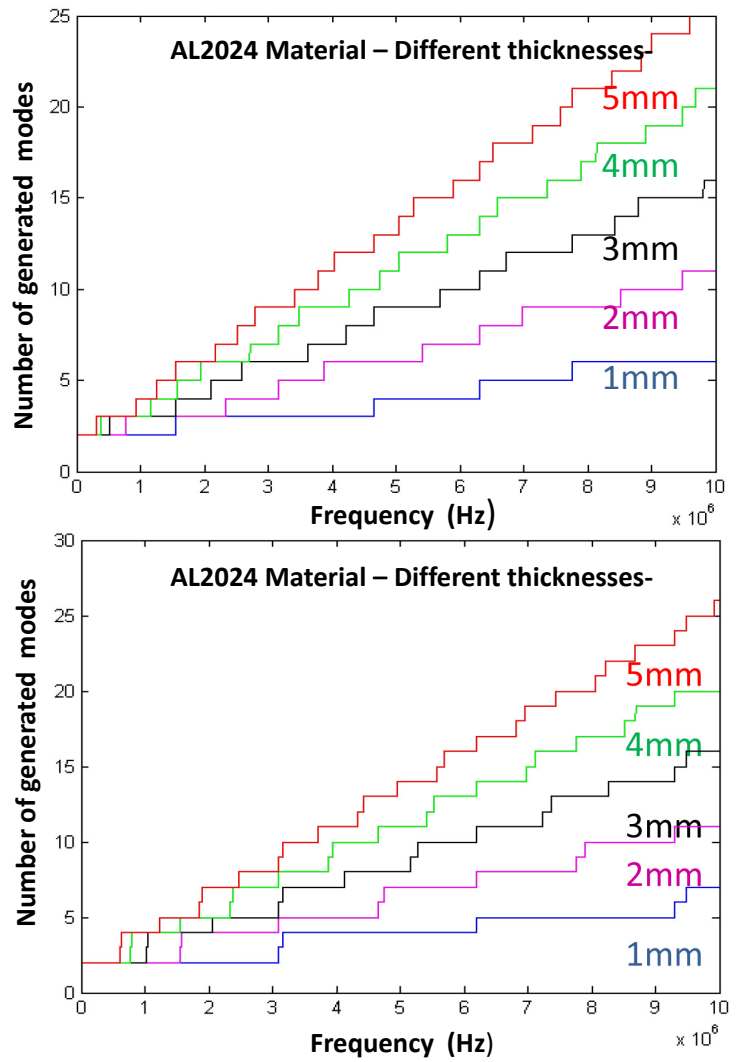

(b)

Figure 4. Number of coexisting Lamb wave modes in the symmetric (a) and anti-symmetric case (b) simulated for different operating frequencies and different thicknesses. [Simulation performed for

$$
\text { AL2024] }
$$

The calculation of the two determinants $(\mathrm{A}, \mathrm{B}),(\mathrm{C}, \mathrm{D})$ yields for isotropic materials to Rayleigh-Lamb characteristic equation.

(a) 


$$
\frac{\tan p d}{\tan q d}=-\left[\frac{4 k^{2} p q}{\left(k^{2}-q^{2}\right)^{2}}\right]^{ \pm 1}
$$

Where, +1 corresponds to the symmetric mode whereas -1 corresponds to the anti-symmetric mode of motion.

This equation allows the calculation of dispersive curves and thus allows the identification of each propagation mode for a fixed operating frequency and a determined structure thickness. According to our diagnosis methodology that consists on a comparison between a healthy and damaged structure's signature, equation (9) will be useful to minimize the number of generated Lamb mode and thus minimize the complexity of the different interactions that may occurs in the structures thickness.

\section{LAMB WAVE GENERATION USING PIEZOELECTRIC SENSORS/ACTUATORS}

\subsection{Piezoelectric sensor/actuator development}

Many techniques are already described in the literature to generate Lamb waves. The most recent efficient techniques use ultrasonic transducers with a couplant (D. N. AlleyneetP. Cawley, 1996) to facilitate the transmission of ultrasonic energy from the transducer into the tested structure.

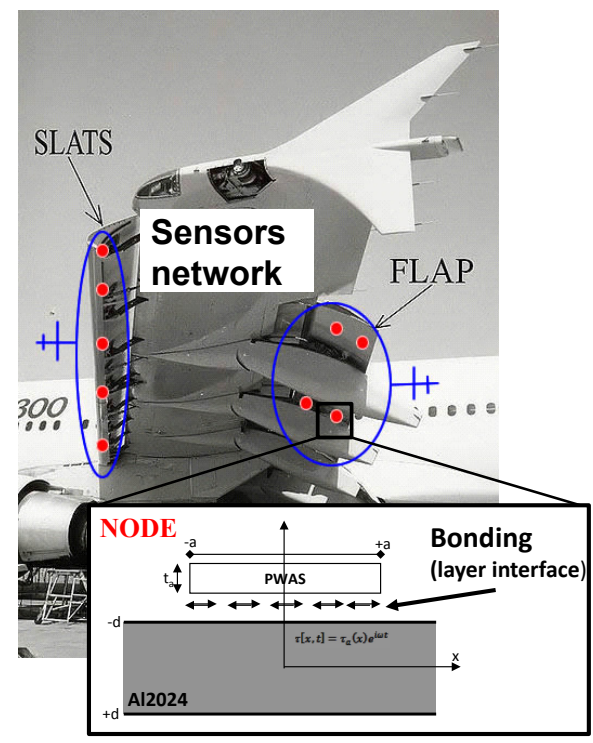

Figure 5 : Airbus A300 left wing. Schematics of the network sensor pasted on specifics mobile parts like the Slats or the kruger flaps. Areas that are often subject to aerodynamics forces and thus subject to damage due to the high cycle of use.
These techniques allow a precise detection of mechanical flaws by a post identification of Lamb modes conversion (Victor Giurgiutiu, 2005) due to the interaction of the generated wave with the flaws. However to reach theirs optimum detection capability, these systems have to generate a pure lamb mode into the structure which is only possible on small areas where the structure's thickness is constant. These techniques are very local and therefore unsuitable for a network integration system intended to aeronautics' structures health monitoring application.

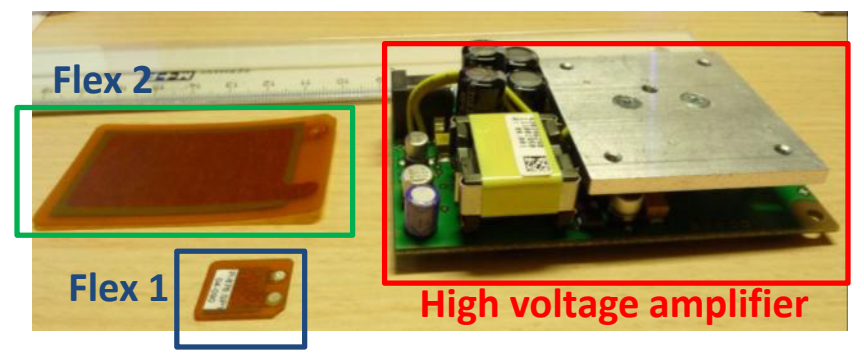

(a)

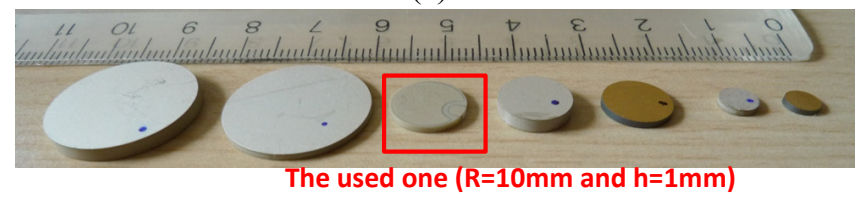

(b)

Figure 6 : (a) DuraAct transducer with its power amplifier (b) PZT bulk transducers.

Therefore, the focus of our research turned on the study of small transducers made of piezoelectric materiel mounted directly onto the surface of the structure within minimal intrusion. The exploitation of the piezoelectric effect allows the generation of a shear stress directly through an interfacing layer into the structure (Victor Giurgiutiu, 2002). To do so, we used two kinds of transducers:

1. PI-DuraAcat P-876.A12 transducer of $61 \times 35 \times 0.5 \mathrm{~mm}$ made from a thin layer of PZT materials staked between two layers of kapton which makes is totally flexible (Cf. Figure 6.a). Because of its capacitive nature and its thin thickness, the transducer needs a high voltage amplifier between $[100 \mathrm{~V}, 400 \mathrm{~V}]$ to have a significant actuation. To perform this amplification we used the E-835 DuraAct ${ }^{\mathrm{TM}}$ Piezo Driver Module that provides $30 \mathrm{~W}$ of power output with a voltage of $250 \mathrm{~V}$ for an input signal of $10 \mathrm{~V}$.

2. Custom transducer made from PZT-5H material designed to have a radial oscillation frequency of 200 $\mathrm{kHz}$ (Cf. Figure 6.b). As explained previously, this frequency minimizes the number of coexisting Lamb 
modes. The used material has a radial coefficient frequency of 1980 Hz.m which fixes the sensor diameter to $\sim 10 \mathrm{~mm}$. In the other hand, the normal mode frequency constant is equal to $2032 \mathrm{~Hz} . \mathrm{m}$. The thickness was therefore fixed to $1 \mathrm{~mm}$ to have a second oscillation mode frequency equal to $2 \mathrm{MHz}$.

As shown in figure 4 , to minimize the number of generated modes into the structure, we should use an excitation frequency that stays below $500 \mathrm{kHz}$. This condition minimizes the number of Lamb modes (Hamza Boukabache et al, 2011) and thus reduces the interference into the host structure which makes the interpretation of the acquired signals much easier. In opposite to what was already described in the literature (Victor Giurgiutiu, 2005), we have chosen to exploit both symmetrical and antisymmetrical modes which is easier to generate than pure modes.

\subsection{Experimental setup}

To demonstrate the generation of Lamb waves into plates likes structures we installed a test bench based (Figure 7 and 8 ) on an National instrument acquisition card that offers up to 8 simultaneous acquisition channel of 14bits resolution and $2.5 \mathrm{MSample} / \mathrm{s}$ speed. In the other hand the stimulus generation is based on an Agilent 3322 arbitrary waveform generation instrument. The communication with the $\mathrm{PC}$ is performed through an USB-VISA protocol which allows us to transfer the stimulus data directly from the data processing software to the instrument.

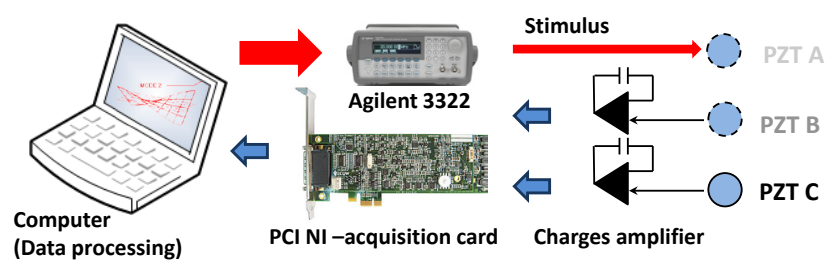

Figure 7: Synoptic of the test bench setup.

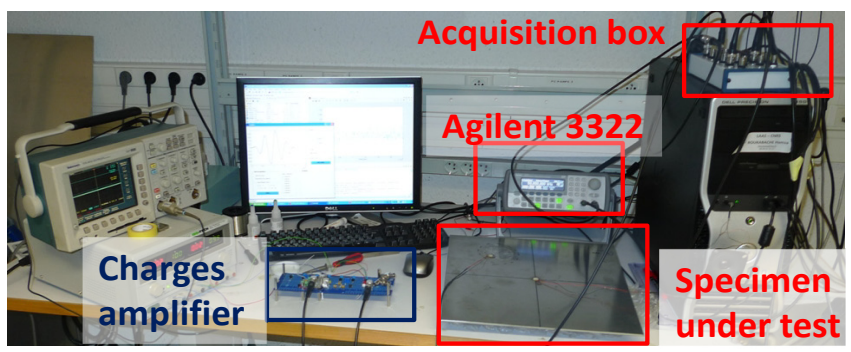

Figure 8: Synoptic of the test bench setup.

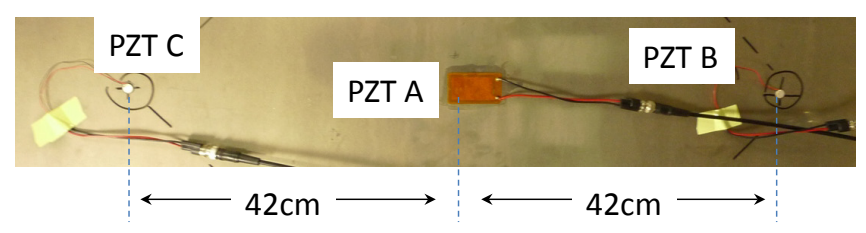

Figure 9: Synoptic of the test bench setup.

The chosen stimulus waveform is the pure tone sine modulated by a Hanning window which is commonly used in the literature (Hamza Boukabache et al, 2011).

In actuation mode, the excitation frequency was fixed to 200 $\mathrm{kHz}$ which minimizes the number of generated mode to 3 into an Al2024 plate of $3 \mathrm{~mm}$ thickness.

When the piezoelectric transducer is used in sensor mode, a charge amplifier based on a capacitive feedback is also necessary to have an exploitable voltage signal. According to piezoelectric effect, when subject to a mechanical stress, the transducer generates electrics chares Q [Coulombs] at its respective electrodes that are not directly exploitable. A capacitance $\mathrm{C}$ [Farads] should be added to perform the voltage conversion $\mathrm{V}=\mathrm{Q} / \mathrm{C}$. According to this equation, when an operational amplifier is used, the capacitive feedback fixes the amplification. A step of signal processing is needed to filter the converted voltage.

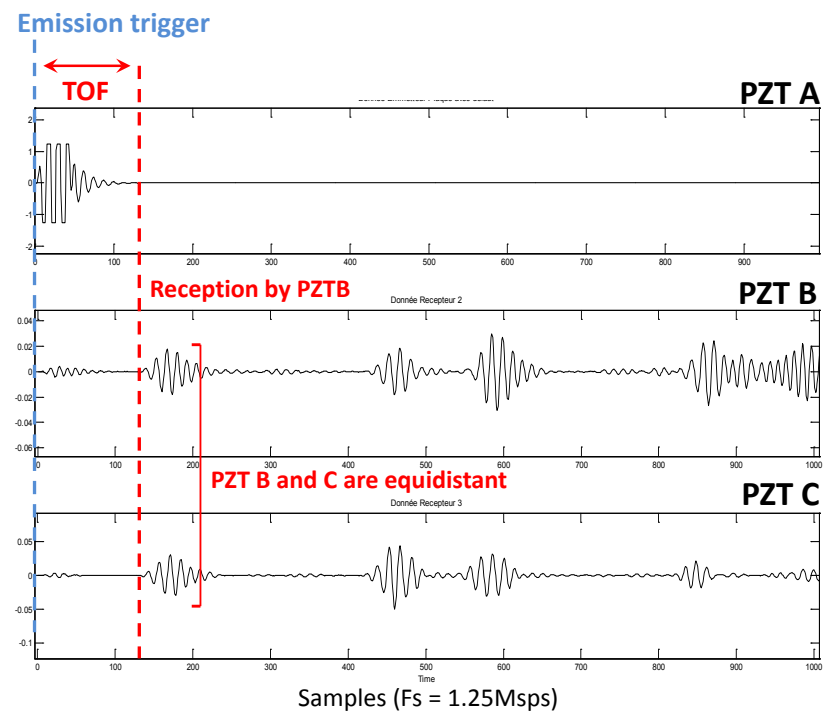

Figure 10 (a) Lamb wave generation into a structure specimen. Note that the time of flight between the emitter and the receptors (TOF) is $112 \mu \mathrm{s}$

Experimental measurements showed that for our operating frequency a simple bandpass filter based on Sallen-Key analog circuit architecture presenting an attenuation factor of $40 \mathrm{~dB}$ and a bandwidth of $10 \mathrm{kHz}$ is enough to get an 
acceptable SNR. Actually this is only true for metallic alloys structures.

From figure 9, notice that the flexible transducer is used as an emitter when the PZT bulk transducers are used in passive sensing mode. As shown in figure 10 the time delay between the emission of PZT A and the reception of PZT B and C gives us the group velocity of the generated wave. This time delay is known as the Time of flight (TOF) of the wave. The 3 transducers are pasted with a distance of $420 \mathrm{~mm}$ from each other. From figure 10, we extract the time of flight which is equal TOF $=112 \mu \mathrm{s}$. For the traveled distance, the wave speed was therefore equal to $4200 \mathrm{~m} / \mathrm{s}$.

\section{DAMAgeS DETECTION :}

In order to detect mechanical flaws in complex structures such as aeronautics flats, wingbox, intra and extra wing panel we developed and algorithm capable to diagnosis and localize damage by comparing the acquired signature to a base line captured before the commissioning of the plane. By minimizing the number of Lamb modes generated into the structure (figure 3) this method circumvents the issues linked to the multiple reflections and the different interactions into the structure.

Globally, there are two methods to detect a defect using guided waves.

- $\quad$ The simplest and the most standard one consist to extract one signature per node using a pulse-echo technique. Using an analogy with sonar world, pulse echo technique consists to emit an impulse and listens to the echoes reflected by the medium where the waves propagate. At final, this configuration gives as many signatures (signals) as the used number of nodes.

- A second more complex technique consists to use an emitter and a receptor. The used principle is based on pitch-catch method. This one consists to detect the deformation and distortions that occurred to the transmitted wave. A baseline is therefore systematically used

\subsection{Crack detection using pulse-echo based NDE technique:}

Inspired from electromagnetic radar world, pulse-echo technique uses at least one node to probe a local area looking for mechanical flaws. The principle is based on the capability of the piezoelectric patch to act both as actuators and as sensors. The node emits a short pulse noted $\mathrm{x}(\mathrm{t})$ given by Eq.9 then it listens for echoes.

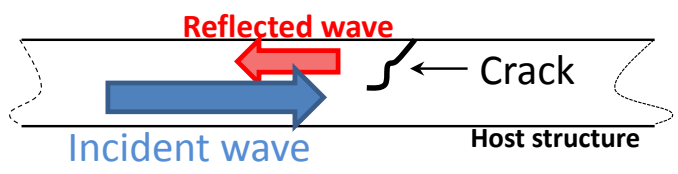

Figure 11. Pulse echo principle

For the simplest case where a pure Lamb mode is generated, the reflected signal could be assumed as a simple image of the emitted one $\mathrm{x}(\mathrm{t})$. In this case we can suppose that the reflected signal $r(t)$ form the crack is only delayed noisy and attenuated.

$$
r(t)=\alpha x(t-D)+\beta(t)
$$

With:

$\alpha:$ Attenuation factor (it depends on the structure nature)

D: [s] Time propagation delay or time of flight (TOF)

$\beta$ : [V] Noise factor

Thus, by knowing the group velocity of the generated pulse $x(t)$ we can use an estimation of the time delay D and calculate the location of the damage.

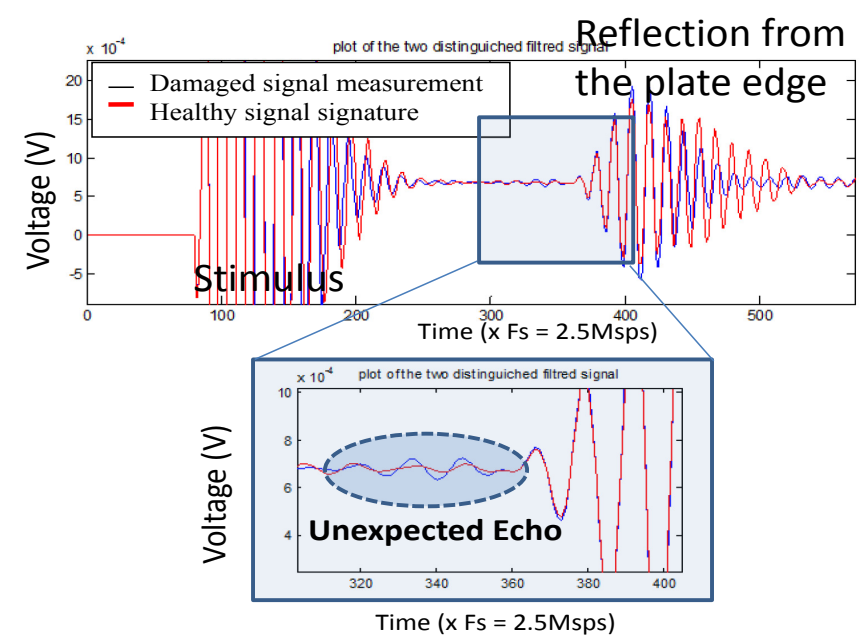

Figure 12. Superposition of the healthy signature and the damaged structure response.

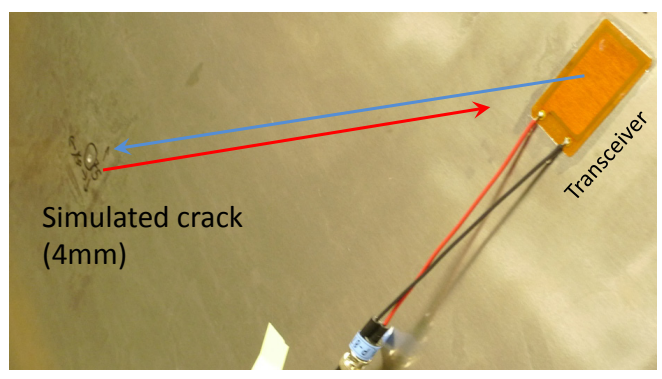

Figure 13. Specimen 1 under test (Crack of $4 \mathrm{~mm})$ 


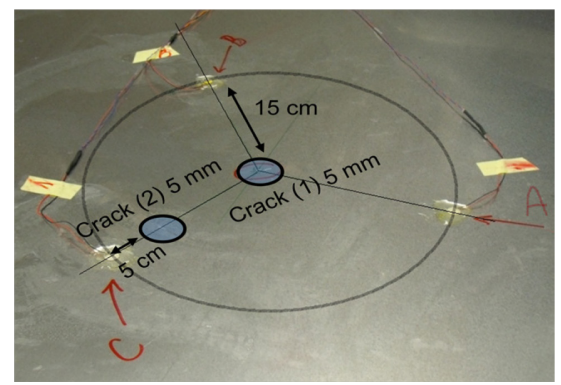

Figure 14. Specimen 2 under test (two cracks of $5 \mathrm{~mm}$ ). The edges of the plate are far away from the sensors (150 $\mathrm{cm})$. This configuration neglects the effect of the edges echoes into the acquired signals.

From figure 12, the crack echo takes $128 \mu$ s to perform a go and back from the transducer to the crack then from the crack to the transducer. We know from the previous experimental results that group velocity is $4200 \mathrm{~m} / \mathrm{s}$ into this material. Therefore, the crack is located at $27 \mathrm{~cm}$ from the transceiver. Using at least 3 nodes we are capable to locate the damage using a basic triangulation algorithm.

\subsection{Crack localization using pulse-echo based NDE technique:}

Inspired from radar world and from the principle presented in figure 11, we created an algorithm based on three sensors capable to locate two cracks using a smart identification of the echoes emitted by the defects.

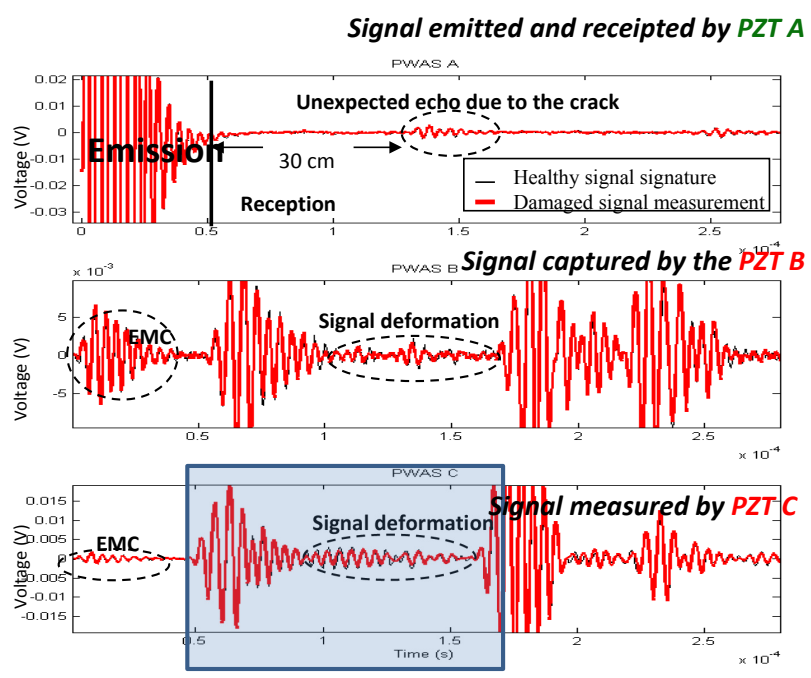

Figure 15. Detection and localization of a crack situated at $15 \mathrm{~cm}$ from sensors $\mathrm{A}, \mathrm{B}$ and $\mathrm{C}$. The results presented are representative of step 1 and 2 .

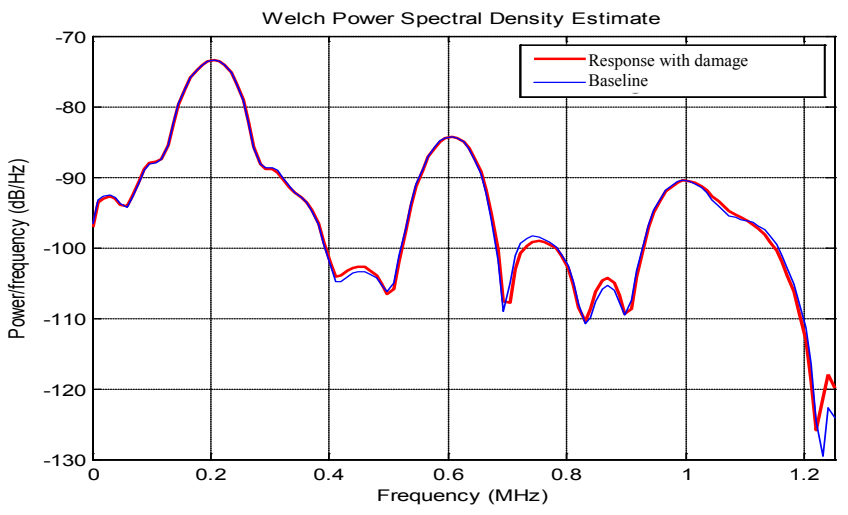

(a)

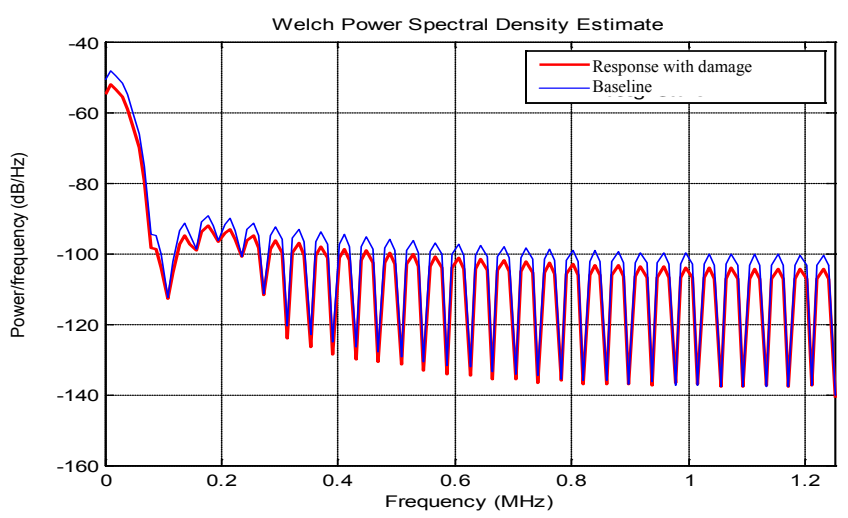

(b)

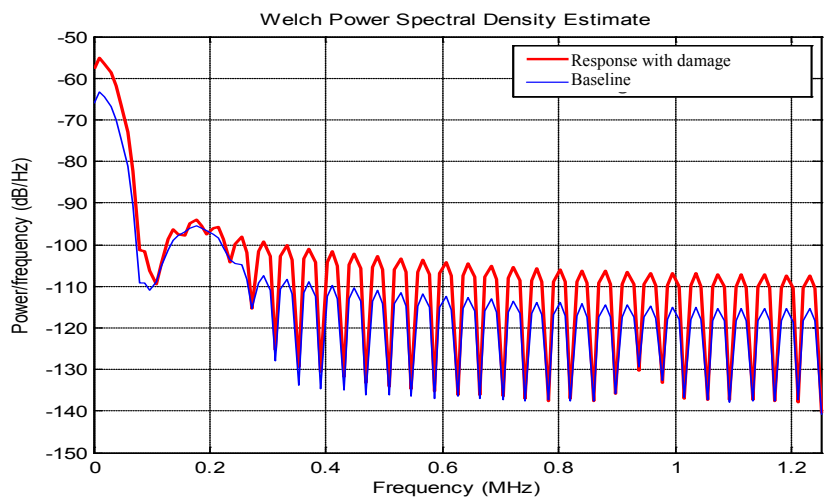

(c)

Figure 16. Power Spectral Density (PSD) analysis of the signals saved when PZT A is configured as an emitter while $\mathrm{B}$ and $\mathrm{C}$ are pure receptors. They are representative of Steps 1 and 2. (a) PSD of the signal acquired by PZT A before and after damage. (b) PSD of the signal acquired by PZT B before and after damage.

(c) PSD of the signal acquired by PZT C before and after damage.

The specimen under test is presented in figure 14. Two cracks were artificially induced between three sensors A, B and $\mathrm{C}$ placed at a circle of $30 \mathrm{~cm}$ diameter. The algorithm is constituted of six steps: 


\begin{tabular}{|l|l|}
\hline Steps & \multicolumn{1}{|c|}{ Algorithm } \\
\hline $\mathbf{1}$ & PZT A emits a impulse (C and B off) \\
\hline $\mathbf{2}$ & Echoes acquisition by PZT A \\
\hline $\mathbf{3}$ & PZT B emits a impulse (A and C off) \\
\hline $\mathbf{4}$ & Echoes acquisition by PZT B \\
\hline $\mathbf{5}$ & PZT C emits a impulse (A and B off) \\
\hline $\mathbf{6}$ & Echoes acquisition by PZT C \\
\hline
\end{tabular}

The execution of all the algorithm's steps brings 9 plots. The case where PZT A is active, B and C are passive is presented in figure 15 and 16 . The figures are respectively an analysis in time domain and in frequency domain.

As expected, the cracks reflect echoes to the emitter PZT A (Figure 15). They also induce deformations to the transmitted signals that are acquired by PZT B and C.

As depicted in figure 16 a simple frequency analysis based on spectral density estimation shows no difference between the different signatures. A small amplitude variation is noticeable in figure 16.b and 16.C at low frequencies. However these variations are too low to indicate the presence of a defect.

To perform the diagnosis we executed a simple signal subtraction between the baseline and the damaged signature.

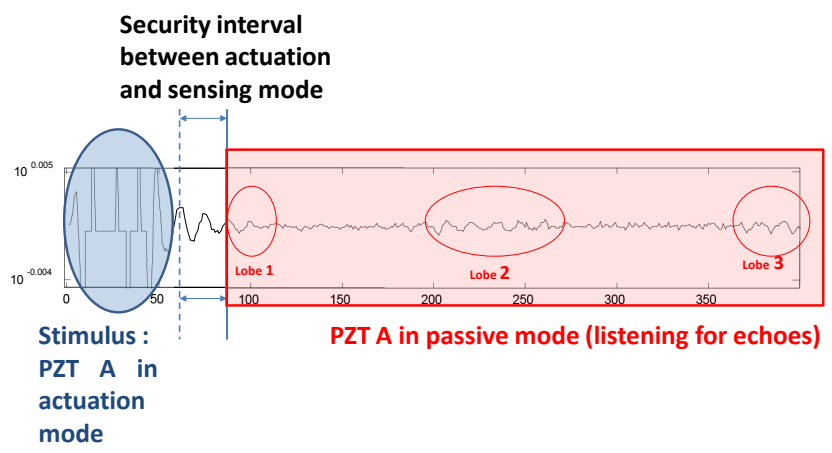

Figure 17: Residue of the temporal subtraction between the healthy and damaged signals acquired by PZT A.

As presented in figure 17, the residue has two operational zones:

- Stimulus area : time interval where the sensor is in actuation mode

- Listening area: interval where the sensor was totally passive.

Between the two zones, we fixed a security interval of $12 \mu \mathrm{s}$. This value was fixed to avoid erroneous detection due to a poor coupling between the structure and the PZT. This interval limits the detection to defects located at more than $50 \mathrm{~mm}$ from the activated sensor.

A joint time frequency analysis (JTFA) based on wavelet transform was applied to the residue of the subtraction (Cf. Figure 18).

It allows us to decompose our reflected signal into both frequency and time domain which make the comparison much accurate.

To perform the continue wavelet transform Eq .11 we used the Morlet wavelet function as the temporal correlation coefficient $\psi_{s, \tau}(t)$.

$$
g(s, \tau)=\int_{-\infty}^{\infty} f(t) \psi_{s, \tau}(t)^{*} d t
$$

With : $f(t)$ the transmitted signal.

The decomposition presented in figure 18.b was performed on the temporal reflected signals captured by PZT A, B and $\mathrm{C}$ for each step of the algorithm.

The figure 18.b represents the percentage of energy for each wavelet coefficient in time-frequency domain. By isolating the $200 \mathrm{kHz}$ coefficients that corresponds to our excitation frequency stimulus we plot the reflectivity profile of this frequency during its travel into the host plate ( $\mathrm{Cf}$ Figure 18.C).
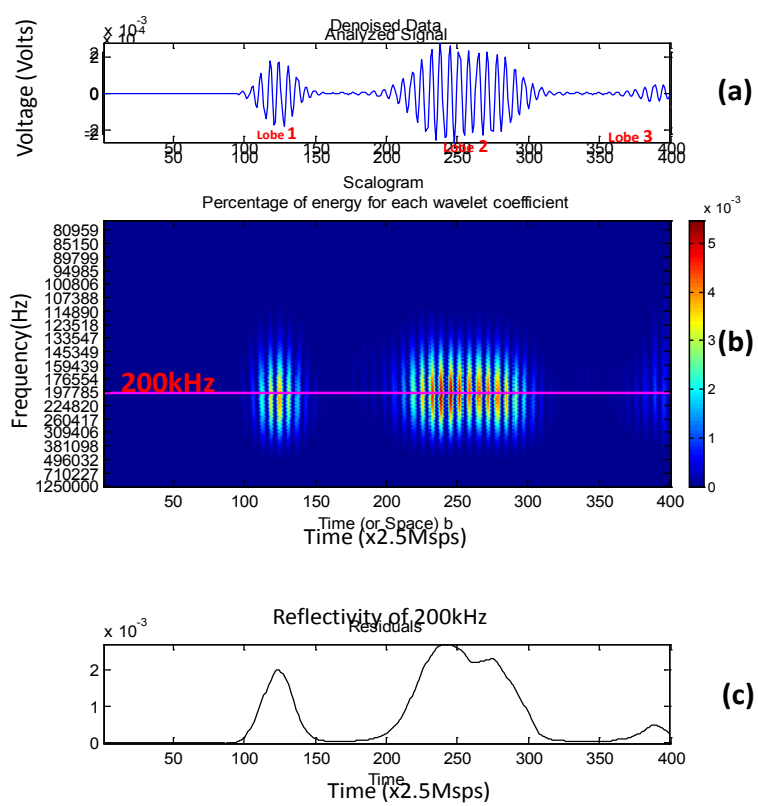

(c)

Figure 18: (a) Reconstructed residue of the temporal subtraction between the healthy and damaged signals acquired by PZT A. (b) Wavelet decomposition of the temporal reflected signal. (c) Extraction of the $200 \mathrm{kHz}$ reflectivity profile. 


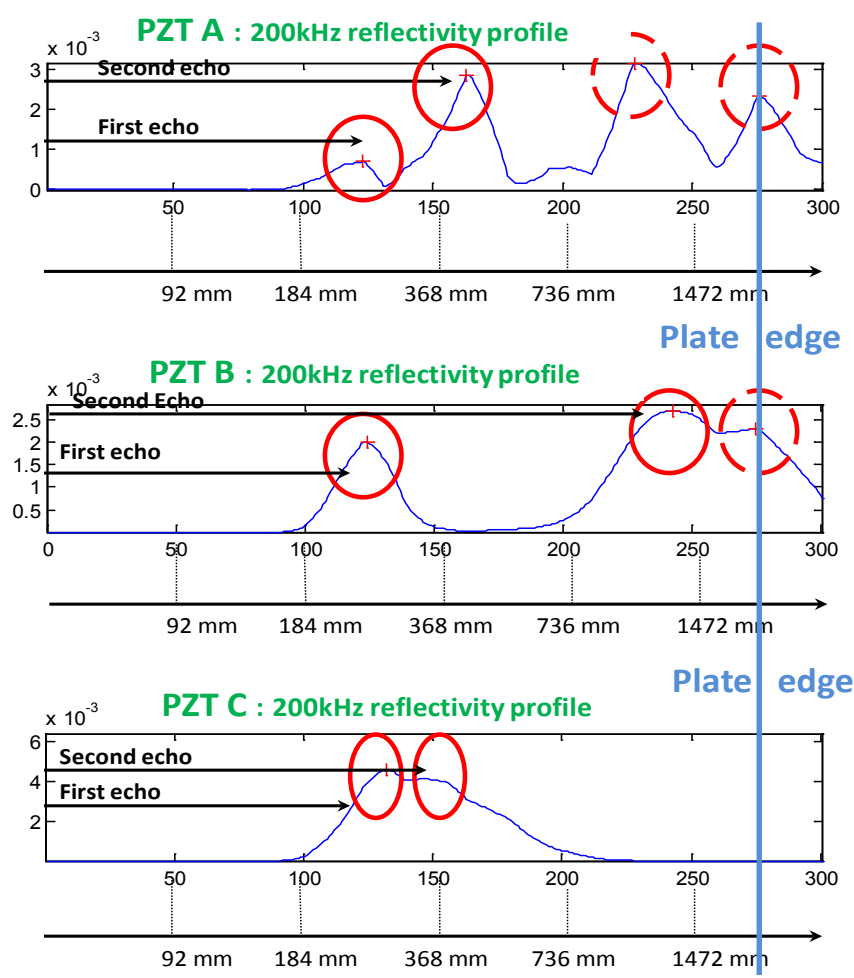

Figure 19: $200 \mathrm{kHz}$ reflectivity profiles for sensors A, B and $\mathrm{C}$ extracted for each steps of the presented Algorithm in table 1.

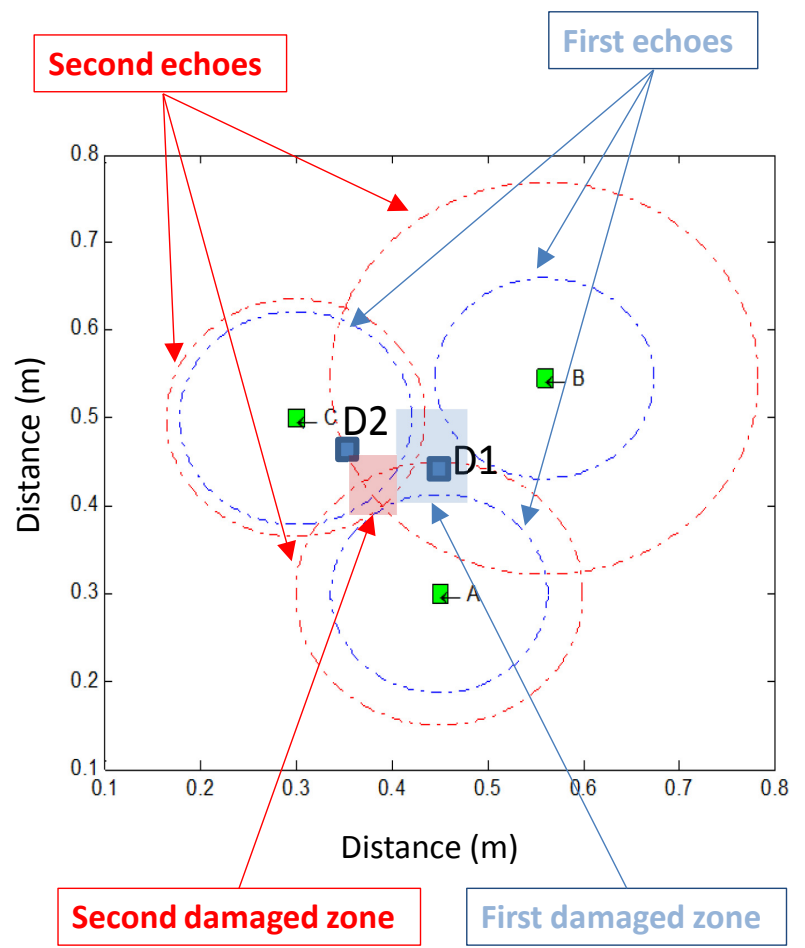

Figure 20: Map of the plate with the location of the sensors $\mathrm{A}, \mathrm{B}$ and $\mathrm{C}$. Triangulation algorithm shows the damaged areas. Notice that D1 is in the middle of the first expected damaged zone. The second crack is located near the second calculated damaged area.

The superposition of the reflectivity profiles for the PZT A (Step 2 of the Algorithm presented in table 1), PZT B (Step 4) and PZT C (Step 6) show many peaks due to unexpected echoes. The fixation of three thresholds respectively $0.7 \mathrm{e}-3$, 1e-3 and 3e-3 for PZT A, B and C allows the application of a simple triangulation algorithm (Cf. Figure 20) based on circles plots. Each peak in figure 19 corresponds to a circle.

The locations of the cracks are deduced by the areas where the circles are close to each others.

\subsection{JTFA method reliability.}

We demonstrate the feasibility of cracks detection into thin plate's structures using a comparison with a baseline. In other words the presented method is sensible to the plate signature. To demonstrate the reliability of the method, we should demonstrate that the JTFA (Joint Time Frequency Analysis) algorithm in insensible to the boundary conditions which makes the structure signature unique.

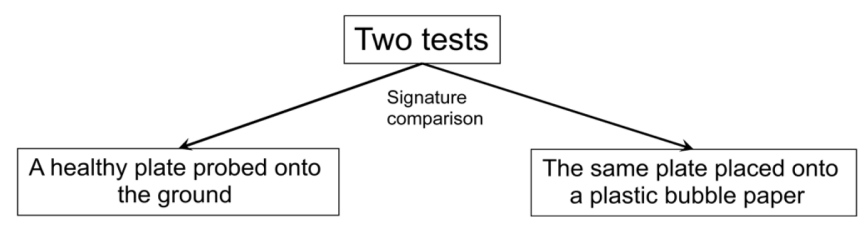

Figure 21: Plan of experience

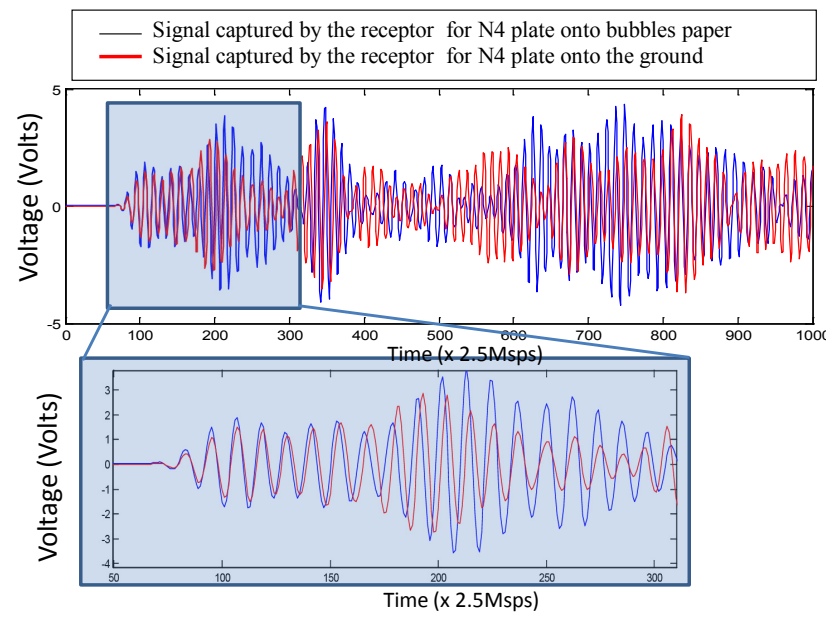

Figure 22: Experimental results. Superposition of the structure's signatures for two different boundaries conditions. 
According to figure 21 we propose using the same test bench presented in figure 7 to extract the signatures of structure N4 (Cf. figure 16) for two cases:

- N4 plate placed onto a solid surface (Ground)

- N4 plate onto a bubble plastic paper.

The results of the experience are presented in figure 22. It shows that the temporal signature seems to be different for the same structure.

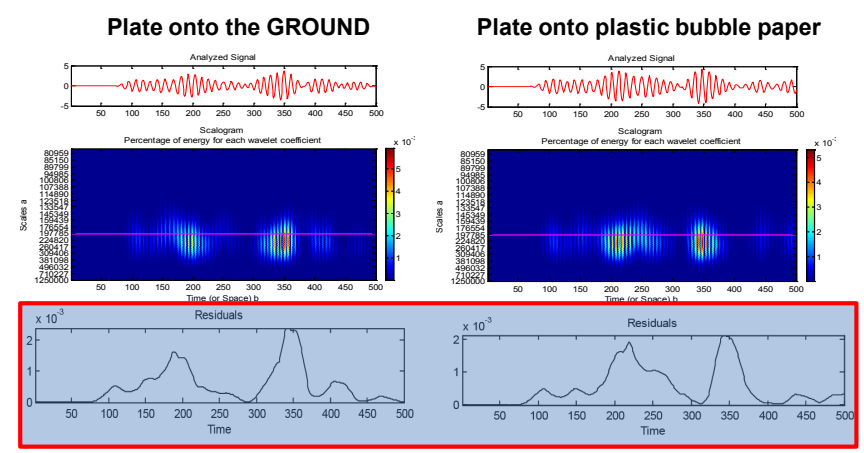

Figure23: Wavelet decomposition. Extraction of the $200 \mathrm{kHz}$ transmitted profile.

The application of the JTFA algorithm on the data presented in figure 23 show that the transmitted $200 \mathrm{kHz}$ signal between the emitter and the receptor is insensible to the boundary conditions.

\subsection{Corrosion detection using pitch catch embedded NDE:}

Corrosion in aircrafts metallic's structures is one of the most common problems that we face during planes life time. It generally appears into the hidden side of the structure where the thickness of the chemical surface processing is thinner.

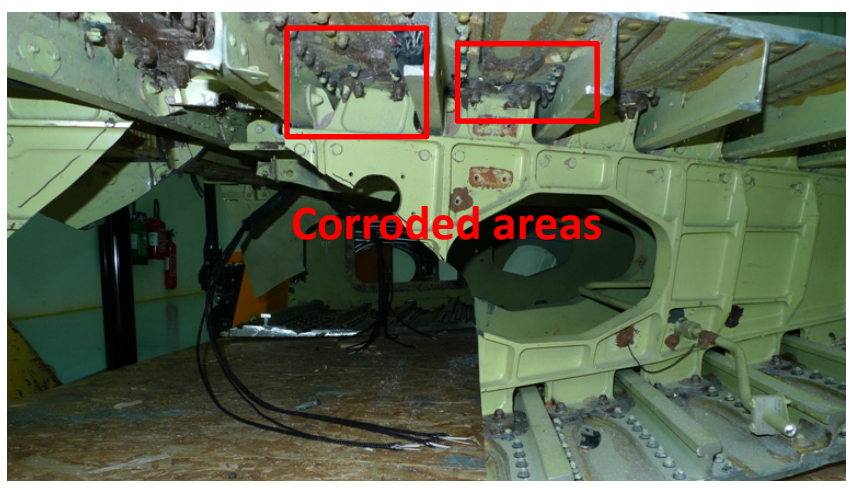

Figure 14. A320 wingbox with corrosion flaw inside the wing. (Acknowledgments to Airbus and EADS-IW)

\begin{tabular}{l|l}
\hline Specimen & Corrosion size (depth- area) \\
\hline $\mathrm{N} 4$ & Baseline (None- None) \\
\hline $\mathrm{N} 1$ & $1 \mu \mathrm{m} \times 230 \mathrm{~cm}^{2}$ \\
\hline $\mathrm{N} 3$ & $1 \mu \mathrm{m} \times 38 \mathrm{~cm}^{2}$ \\
\hline $\mathrm{N} 5$ & $500 \mu \mathrm{m} \times 56 \mathrm{~cm}^{2}$ \\
\hline $\mathrm{N} 6$ & $1 \mathrm{~mm} \times 4 \mathrm{~cm}^{2}$ \\
\hline
\end{tabular}

Table 2. Specimens characteristics
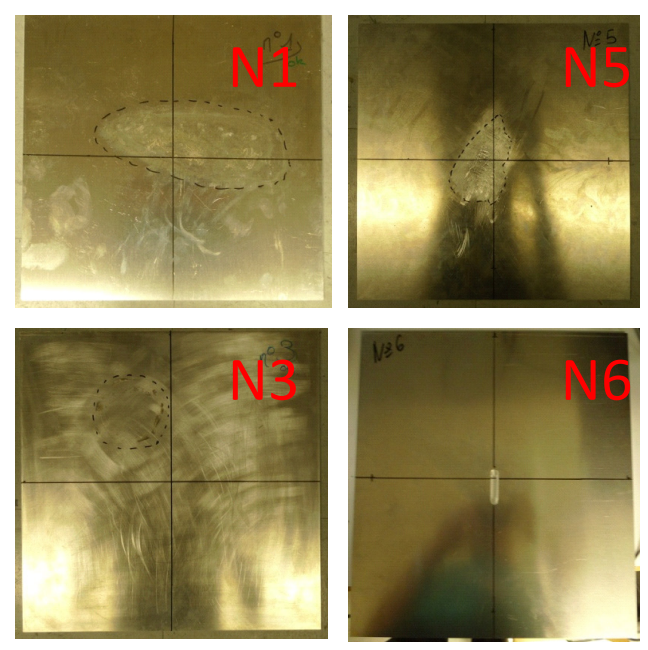

Figure 15. Photos of the corroded specimens (Acknowledgements to CIRMIAT's team who introduced the corrosions onto the specimens)

Therefore the corroded areas are hardly accessible to human beings which make their detection very difficult. Furthermore, the corrosion in Al2024 implies a thickness loss for the structure which weakens its mechanical proprieties.

In order to detect corrosion into aeronautics Al2024 structures, we extracted healthy panels of $32 \mathrm{~cm}$ x $32 \mathrm{~cm}$ x 3 $\mathrm{mm}$ that do not contain rivets or fasteners. We introduced artificially corrosion using a $\mathrm{NaCl}$ attack and thermal processing (Cf. Figure 14). The 5 specimens are listed in the table 2 .

As shown in figure 16, pitch catch technique uses at least two nodes, one as an emitter and the second one as a simple passive sensor (the receptor). For a simple structure as shown in figure 15 the reflected signal captured by the first node contains the damage's echo $x(t-D)$ plus the parasitic echoes due to geometry of the structure. However, in opposite to the crack damage case, the produced echoes are 
very weak in the corrosion case. In fact the corrosion produces a thickness loss that is very small (up to $10 \%$ of the global thickness) which make the reflected echo negligible. In the second hand the receptor sensor captures the transmitted signal noted $T(t)$

$$
T(t)=\gamma x(t+\Delta T)+\Sigma_{i}\left(\alpha_{i} x\left(t_{i}\right)\right)+\beta(t)
$$

With:

$\alpha_{\mathrm{i}}:$ Attenuation factor

$\mathrm{D}_{\mathrm{i}}:[\mathrm{s}]$ Time propagation delay

$\beta$ : $[\mathrm{V}]$ Noise factor

$\gamma:$ Transmission deformation
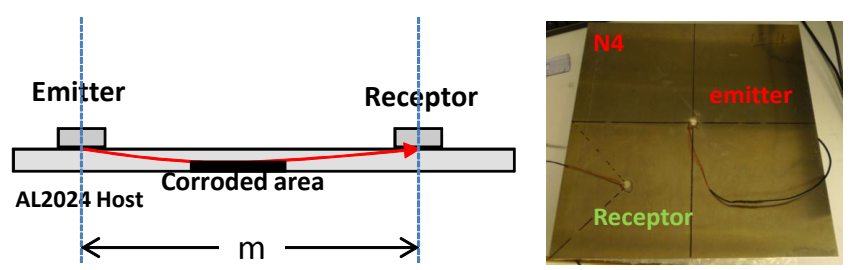

Figure 16. Pitch catch principle

The comparison between the different transmitted signals allows us to detect a corroded zone using a reference or a baseline acquired when the structure was healthy.

To perform the comparision we used the JTFA diagnosis presented previously.

The decompostision presented in figure 17 was performed on the temporal transmited signal captured by the receptor for the healthy structure (specimen N4 presented in figure 16). The figure 17.b presents the percentage of energy for each wavelet coeficient in time-frequency domaine. By isolating the $200 \mathrm{kHz}$ coeficients that corresponds to our excitation frequency we plot the transmission profile of this frequency during its travel into the host plate.

Figure 18 shows the decompoition of signal N1. Notice the clear difference with the baseline (Cf. Figure 17).

For the plate structure N1 with a corroded area of $230 \mathrm{~cm}^{2}$ with a thickness loss of $1 \mu \mathrm{m}$, the transmitted wave had during its travel a different intetergaction with the host structure than for N4 (Baseline)

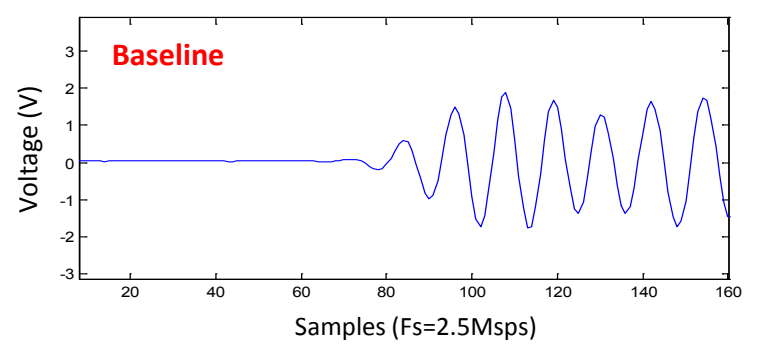

(a)
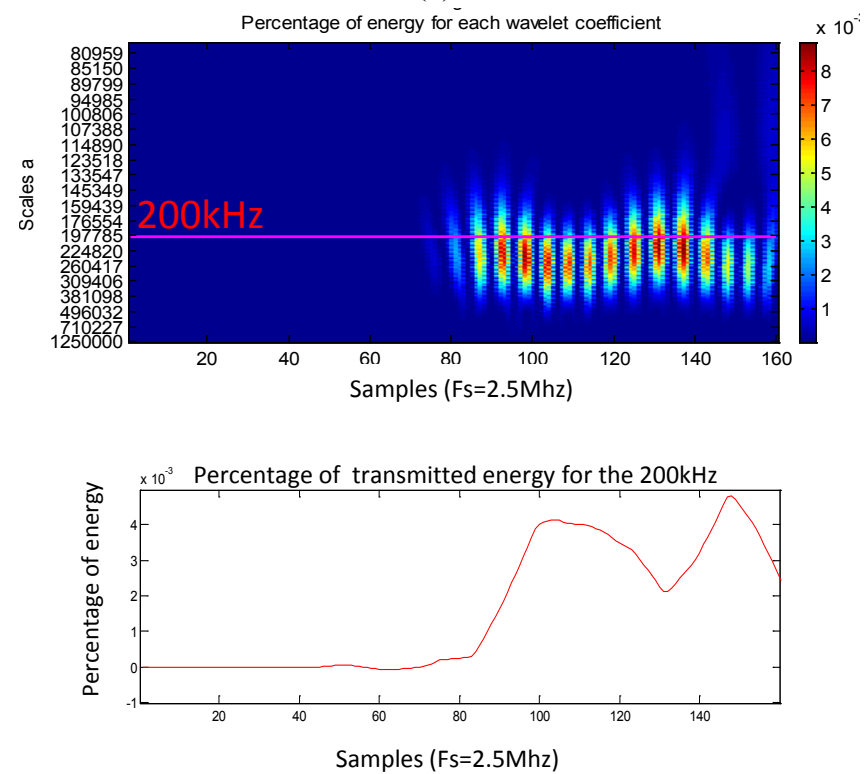

(b)

Figure 17. (a) The acquired transmitted signal for the witness specimen N4. (b) Wavelet decomposition of the temporal transmitted signal. We extract the profile of propagation of the $200 \mathrm{kHz}$ frequency

From figure 19 it is clear that each specimen plate has its own signature. The measurements were performed 3 times for each plate using the same measurements conditions.

Notice that the more the corroded area is large the more is the amplitude of the first peak. The depth of the thickness loss seems to have also a direct link with the amplitude.

For the extreme case where the thickness loss is $1 \mathrm{~mm}$ the amplitude of the transmitted signal is significantly low in opposite to all the other signals. Due to the high thickness loss $(33 \%)$, a possible explanation would be the fact that most of the wave energy emitted by the actuator is reflected by the damage and only a low amount of energy is transmitted. 


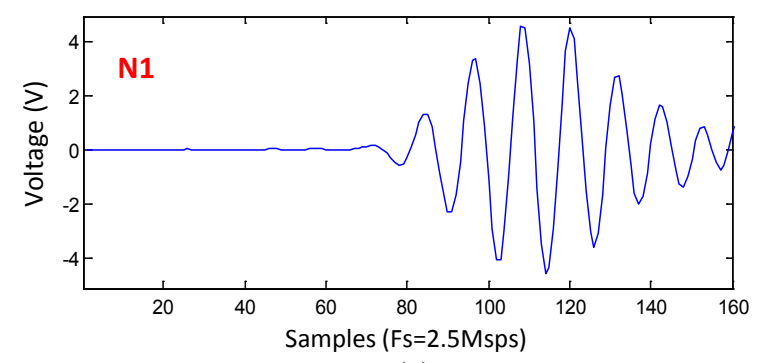

(a)
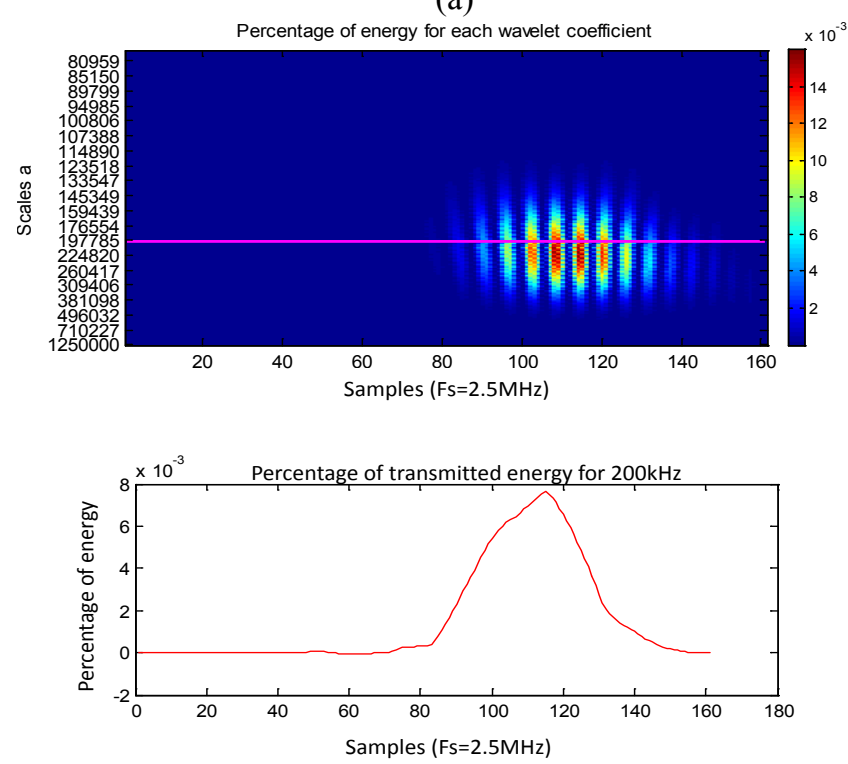

(b)

Figure 18. (a) The acquired transmitted signal for the specimen N1. (b) Wavelet decomposition of the temporal transmitted signal. We extract the profile of propagation of the $200 \mathrm{kHz}$ frequency

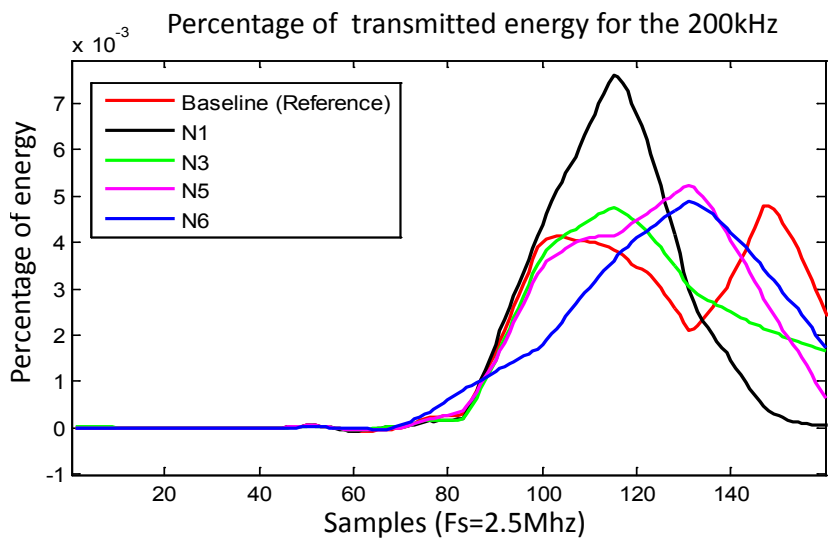

Figure 19. Superposition between the different transmissions signals for the different specimens

The superposition of the different transmitted profile (Cf. Figure 19) allows us to diagnosis the presence of corrosion into the structure. However, unlike pulse echo technique our methodology is unable to locate the corrosion. We only know that the corroded area is situated between the emitter and the receptor. Therefore the resolution of this method is totally linked to the distance between the transducer and thus to the fixed meshing.

\section{Conclusion}

In this paper we demonstrate the feasibility of crack localization using a simple algorithm and corrosion detection inside A12024 structures using a joint time frequency analysis.

The next step of our research is the hardware and software integration to make the nodes autonomous. In parallel, electromechanical impedance based diagnosis is studied and is currently integrated into a reconfigurable system on chip. The idea is to couple two techniques, a very local one associated with a medium range inspection techniques based on guided waves. The current development is also tested for carbon composite structures to localize delamination or fiber disarrangements.

\section{ACKNOWLEDGEMENT}

This work is done in the frame of DIAGNOSTAT project which is funded by the Midi Pyrenees council and the Ministry of Industry. Many thanks to EADS-IW and Airbus group who provided the aircrafts specimens.

\section{REFERENCES}

Henry A. Sodano, (2007). Development of an Automated Eddy Current Structural Health Monitoring Technique with an Extended Sensing Region for Corrosion Detection, Structural Health Monitoring vol. 6 no. 2 111-119

Liang, C (1994). Coupled electro-mechanical analysis of adaptive material system-determination of the actuator power consumption and system energy transfer. J. Intel. Mater. Syst. Struct., 5, 12-20.

Sun, F.P. (1995). Automated real-time structure health monitoring via signature pattern recognition. Proc. SPIE, 2443, 236-247.

Boukabache, Hamza et al (2012). "System-on-Chip Integration of a New Electromechanical Impedance Calculation Method for Aircraft Structure Health Monitoring." Sensors 12, no. 10: 13617-13635.

Victor Giurgiutiu, (2004) Embedded-ultrasonics Structural Radar for In Situ Structural Health Monitoring of Thinwall Structures, Structural Health Monitoring June 2004 vol. 3 no. 2 121-140 
McIntosh, Greg, (1996) Infrared thermography monitors composite consolidation, Advanced Materials \& Processes;Dec96, Vol. 150 Issue 6, p29

Salsabil Ksouri, Mouhamed Matmat, Hamza Boukabache (2011); Damage detection in composite laminates aeronautics structures through accelerometers network, Advances in Materials Sciences ; Volume 11, Number 2 p37-43

Dixon, S et al. (2004). Inspection of rail track head surfaces using electromagnetic acoustic transducers (EMATS). Insight - Non-Destructive Testing and Condition Monitoring, Volume 46, Number 6,

OLYMPUS, (2009). Flaw Detectors, BondMaster, http://www.olympus-ms.com/en/bondmaster1000eplus/

Seth S. Kessler et al (2009) "A Cable-Free Digital SensorBus for Structural Health Monitoring of Large Area Composite Structures" Proceeding of the Annual Conference of the Prognostics and Health Management Society, San Diego 2009

Victor Giurgiutiu, (2005). Tuned Lamb Wave Excitation and Detection with Piezoelectric Wafer Active Sensors for Structural Health Monitoring, Journal of Intelligent Material Systems and Structures vol. 16 no. 4 291-305

Hamza Boukabache et al, (2011) piezoelectric wafer active sensor network for aircraft structures damage localisation: pitch-catch method. International Workshop on Structural Health Monitoring (IWSHM 2011), Stanford (USA), 13-15 Septembre 2011, pp.555561

Hamza Boukabache et al, (2011) "Sensors/actuators network development for aeronautics structure health monitoring", Proceeding of IEEE sensors, Limerick p1157-1160

Hamza Boukabache et al, (2012) "Structural Health Monitoring on Metallic Aircrafts Using Flexible and Bulk PZT Transducers: Case of Corrosion Detection and Crack Localization" Proceeding of the Annual Conference of the Prognostics and Health Management Society, Mineapolis 2012

D. N. AlleyneetP. Cawley, (1996), The excitation of Lamb waves in pipes using dry-coupled piezoelectric transducers. , Volume 15, Number 1 (1996), 11-20, DOI: $10.1007 / \mathrm{BF} 00733822$

Victor Giurgiutiu, (2002) Lamb Wave Generation with Piezoelectric Wafer Active Sensors for Structural Health Monitoring. Annual International Symposium on Smart Structures and Materials and 8th Annual International Symposium on NDE for Health Monitoring and Diagnostics, 2-6 March 2002, San Diego, CA

Dustin Thomas et al, (2004) Corrosion Damage Detection with Piezoelectric wafer Active Sensors. Annual International Symposium on Smart Structures and Materials and 9th Annual International Symposium on NDE for Health Monitoring and Diagnostics, 14-18 March 2004, San Diego, CA

\section{BIOGRAPHIES:}

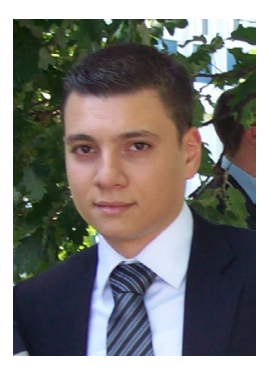

Hamza BOUKABACHE received his M.S in embedded aerospace electronics from the French national institute of applied science at Toulouse (INSA) in 2009 and the same year he got a Master research in micro and nano-systems from Paul Sabatier University at Toulouse. He is currently preparing his Ph.D. with the French national center for scientific research and Airbus FRANCE. Current research interests include Aerospace structure health monitoring using wide sensors network, energy harvesting and reconfigurable electronics development.

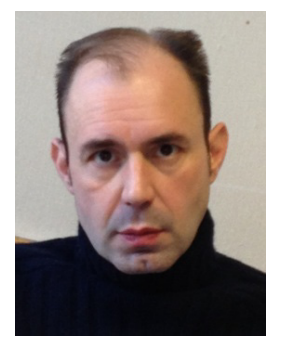

Christophe Escriba is an associate Professor at Department of Electrical Engineering, INSA Engineering University in Toulouse and active researcher at LAAS-CNRS. His research field are micro electronics, micro fluidics and Structural health monitoring using sensors networks

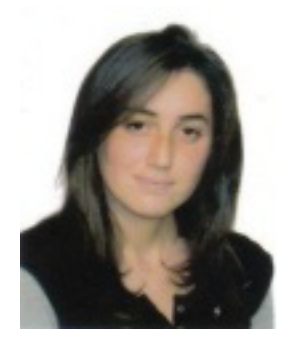

Sabeha Fettouma ZEDEK is a $\mathrm{PhD}$ student at LAAS-CNRS. She received a Master of engineering in Electronics in 2009 from university of Alger, Algeria followed by a Master of Science in micro and nano systems from Paul Sabatier University at Toulouse, France. Her research topics include aerospace SHM and reconfigurable hardware applied to multi sensors instrumentation.

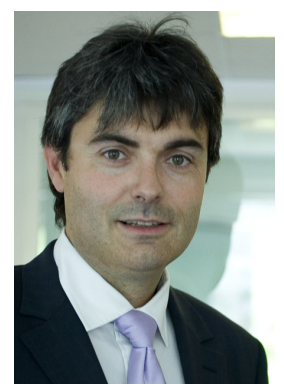

and packaging.
Jean-Yves FOURNIOLS is Full Professor at Department of Electrical Engineering, INSA Engineering University in Toulouse and director of Nano Engineering and Integration Systems research (N2IS) group, at LAAS-CNRS. His research field of interest are sensor networks dedicated to structural health monitoring based on advanced Microsystems assembly 